\title{
First-principles Study on the Adsorption Properties of Phenylalanine on Carbon Graphitic Structures
}

\author{
[J. Korean Phys. Soc. 67, L2020 (2015)]
}

\author{
Seoung-Hun KAng, Dae-Gyeon Kwon, ${ }^{*}$ Sora PARK ${ }^{\dagger}$ and Young-Kyun Kwon ${ }^{\ddagger}$ \\ Department of Physics and Research Institute for Basic Sciences, Kyung Hee University, Seoul 02447, Korea
}

DOI: $10.3938 / \mathrm{jkps} .68 .374$

The electronic supplementary material is missing in this article.

The online version of this article (DOI: 10.3938/jkps.67.2020) contains electronic supplementary materials. 